\title{
A repercussão psicossocial da mastectomia para a mulher
}

\author{
The psychosocial impact of mastectomy for women \\ El impacto psicosocial de la mastectomía para las mujeres
}

Recebido: 11/06/2021 | Revisado: 21/06/2021 | Aceito: 25/06/2021 | Publicado: 10/07/2021

\author{
Maria Linete Monção da Silva Mascarenha \\ ORCID: https://orcid.org/0000-0001-8347-2369 \\ Faculdade de Ensino Superior do Piauí, Brasil \\ E-mail: moncaolinete@gmail.com \\ Gislene Mariana Pereira Castelo Branco \\ ORCID: https://orcid.org/0000-0003-4393-771X \\ Faculdade de Ensino Superior do Piauí, Brasil \\ E-mail: gi.marianna@hotmail.com \\ Ruth Raquel Soares de Farias \\ ORCID: https://orcid.org/0000-0002-0988-0900 \\ Faculdade de Ensino Superior do Piauí, Brasil \\ E-mail: ruthraquelsf@gmail.com
}

\begin{abstract}
Resumo
O câncer de mama ainda é uma doença repleta de estigmas e apresenta diversas situações de ameaça às suas vítimas, afetando a qualidade de vida dessas. Há casos em que o câncer se encontra em um estágio avançado, sendo necessária a utilização da mastectomia, uma cirurgia radical e, portanto, mutiladora, ocasionam transformações dolorosas na vida das mulheres, como alterações da auto-imagem, da auto-estima e comprometimento da sexualidade. Analisar as repercussões psicossociais para a mulher após a mastectomia. Trata-se de uma revisão integrativa da literatura, sendo utilizando como base de pesquisas Sciello, Medline e LILACS, publicados no período de 2015 a 2020, como critérios de inclusão as pesquisas que tratam especificamente sobre o tema, nos idiomas: inglês e português, excluídos os artigos cujo tema não é pertinente a pesquisa, ou resultados duplicados. Os dados encontrados foram analisados, comparados e discutido. A partir do levantamento realizado nos 27 artigos encontrados na base de dados Medline, Scielo e Lilacs, foram excluídos 05 estudos e para esta pesquisa foram utilizados 22 estudos, sendo 07 relacionadas ao primeiro objetivo específico, 09 relacionadas ao segundo objetivo específico e 06 ao terceiro objetivo específico desta pesquisa.
\end{abstract}

Palavras-chave: Mamografia; Mastectomia; Psicossocial; Mulheres.

\begin{abstract}
Breast cancer is still a disease full of stigmas and presents several threatening situations for its victims, affecting their quality of life. There are cases in which cancer is at an advanced stage, requiring the use of mastectomy, a radical and, therefore, mutilating surgery, causing painful changes in women's lives, such as changes in self-image, self-esteem and commitment of sexuality. To analyze the psychosocial repercussions for women after mastectomy. This is an integrative literature review, using as a basis for research Sciello, Medline and LILACS, published in the period 20152020, as inclusion criteria for research specifically dealing with the topic, in languages: English and Portuguese, excluding articles whose theme is not relevant to the research, or duplicate results. The data found were analyzed, compared and discussed. From the survey carried out on the 27 articles found in the Medline, Scielo and Lilacs database, 05 studies were excluded and for this research 22 studies were used, being 07 related to the first specific objective, 09 related to the second specific objective and 06 to the third specific objective of this research.
\end{abstract}

Keywords: Mammography; Mastectomy; Psychosocial; Women.

\section{Resumen}

El cáncer de mama sigue siendo una enfermedad llena de estigmas y presenta varias situaciones de amenaza para sus víctimas, afectando su calidad de vida. Hay casos en los que el cáncer se encuentra en un estadio avanzado, requiriendo el uso de la mastectomía, una cirugía radical y, por tanto, mutiladora, provocando cambios dolorosos en la vida de las mujeres, como cambios en la autoimagen, la autoestima y el compromiso de la sexualidad. Analizar las repercusiones psicosociales para las mujeres tras la mastectomía. Metodología: Se trata de una revisión integradora de la literatura, utilizando como base para la investigación Sciello, Medline y LILACS, publicados en el período 20152020, como criterios de inclusión para investigaciones que aborden específicamente el tema, en idiomas: inglés y portugués, excluyendo artículos cuyo El tema no es relevante para la investigación os resultados duplicados. Los datos encontrados fueron analizados, comparados y discutidos. De la encuesta realizada sobre los 27 artículos encontrados en la base de datos Medline, Scielo y Lilacs, se excluyeron 05 estudios y para esta investigación se 
utilizaron 22 estudios, siendo 07 relacionados con el primer objetivo específico, 09 relacionados con el segundo objetivo específico. y 06 al tercer objetivo específico de esta investigación.

Palabras clave: Mamografía; Mastectomía; Psicosocial; Mujeres.

\section{Introdução}

O câncer de mama ainda é uma doença repleta de estigmas e apresenta diversas situações de ameaça às suas vítimas, afetando a qualidade de vida dessas mulheres. Qualidade de vida é um termo que inclui uma variedade potencial de condições que envolvem a percepção do indivíduo, seus sentimentos e comportamentos relacionados ao seu funcionamento diário, incluindo a sua condição de saúde e intervenções médicas. (Huguet et al., 2019).

Obter o parecer de câncer de mama é uma notícia destruidora, ocasionando grande impacto na vida das pessoas. A paciente e sua família são abarrotados por emoções como sofrimento, medo, raiva, angústia e ansiedade, além de prejuízos nas capacidades sociais, funcionais e vocacionais (Almeida, 2016).

O tratamento utilizado dependerá da extensão da doença e seus atributos. Portanto, após a classificação do câncer de mama, define-se a forma de tratamento a ser desenvolvido. Entre as várias opções de tratamento disponíveis encontram-se a quimioterapia, a radioterapia, a terapia hormonal e a cirurgia, que podem ser administrados individual ou concomitantemente. Se for acertado que há indispensabilidade de se realizar uma cirurgia para a retirada do nódulo, capaz de ser conservadora, a quadrantectomia, na qual é retirada apenas uma parte da mama, sendo necessário ainda a utilização da radioterapia. Contudo, há casos em que o câncer se encontra em um estágio avançado, sendo necessário a utilização da mastectomia, uma cirurgia radical e, portanto, mutiladora. (Unifes, 2002).

A mastectomia ainda é uma das intervenções em que uma maior parte das mulheres com câncer é submetida. É uma mediação temida e que, por ser parte do recurso terapêutico, interfere no estado físico, emocional e social, sucede na mutilação de uma região do corpo que desperta libido e desejo sexual. Esse método interfere na sexualidade, na autoimagem e na estética feminina, na atualidade muito valorizada e ressaltada. (Pinho, 2007).

Tais cirurgias, ocasionam transformações dolorosas na vida das mulheres, como mudanças da autoimagem, da autoestima e comprometimento da sexualidade, visto que a mama é um órgão repleto de simbolismo para a mulher feminilidade, sexualidade e maternidade. A amputação de tal membro pode deixar a mulher envergonhada, mutilada e sexualmente repulsiva, carregando fortes repercussões emocionais. O autoconceito encontrasse afetado devido as alterações na imagem corporal e das modificações devastadoras na aparência física e função (Sampaio, 2006).

O avanço das técnicas de cirurgia plástica nos últimos anos tem proporcionado resultados satisfatórios para a expectativa estética e psicológica da mulher, ao reduzir o trauma causado pela mutilação. (Duarte; Andrade, 2002).

Criada em 2013, a Lei $\mathrm{n}^{\mathrm{o}} 12.802$ prevê que pacientes submetidas a mastectomia têm o direito de realizar a reconstrução mamária por meio do Sistema Único de Saúde (SUS) imediatamente após a retirada do tumor, mas ainda não surte o efeito desejado no Brasil. Segundo o DATASUS, a proporção nos últimos cinco anos é de apenas uma cirurgia de reconstrução a cada 7,5 mastectomias realizadas.

O presente estudo tem como objetivo analisar as repercussões psicossociais para a mulher após a mastectomia, bem como apontar as relações psicossociais das mulheres mastectomizadas e verificar as relações afetivas pós mastectomia. Se mostrando de suma importância a contribuição do psicólogo durante e após o tratamento.

Portanto, é de suma importância relatar na literatura as perspectivas e as repercussões psicológicas na mulher mastectomizada diante dos vários aspectos inseridos na sua vivência, como relacionamento familiar, funcionamento social, imagem corporal, sexualidade e qualidade de vida, assim ajudando o paciente a compreender melhor seu processo e possibilitando uma melhor atuação do psicólogo no tratamento de pacientes com câncer de mama. 


\section{Metodologia}

Refere-se a uma revisão integrativa da literatura, pesquisa de caráter qualitativo (Estrela, C. 2018), relatando o impacto dos efeitos psicológicos e/ou sociais ocasionados em mulheres mastectomizadas após o câncer de mama.

Os dados foram coletados a partir de publicações já elaborados, sendo utilizando como base de pesquisas Sciello, Medline e Lilacs, onde foi utilizado como descritores: câncer de mama, mastectomia, efeitos psicológicos e mulheres mastectomizadas. A questão de pesquisa que norteou a elaboração da presente revisão literária consiste em: "Como o profissional de psicologia pode compreender e contribuir durante a repercussão psicossocial após a mastectomia”?

Foram selecionadas como critérios de inclusão as pesquisas que tratam especificamente sobre o tema, nos idiomas: inglês e português, com um corte entre os anos de 2015 a 2020, que se encontra disponíveis gratuitamente na plataforma. Os critérios de exclusão são as publicações que não condiz com a temática pesquisada e que não se encontra disponíveis gratuitamente na plataforma.

\section{Resultados e Discussão}

A partir do levantamento realizado nos 27 artigos encontrados na base de dados Medline, Scielo e Lilacs, foram excluídos 05 estudos e para esta pesquisa foram utilizados 22 estudos, sendo 07 relacionadas ao primeiro objetivo específico, 09 relacionadas ao segundo objetivo específico e 06 ao terceiro objetivo específico desta pesquisa, conforme fluxograma a seguir.

Figura 1 - Esquema do processo de busca dos artigos da revisão integrativa, aplicação dos critérios de inclusão e exclusão e composição da amostra.

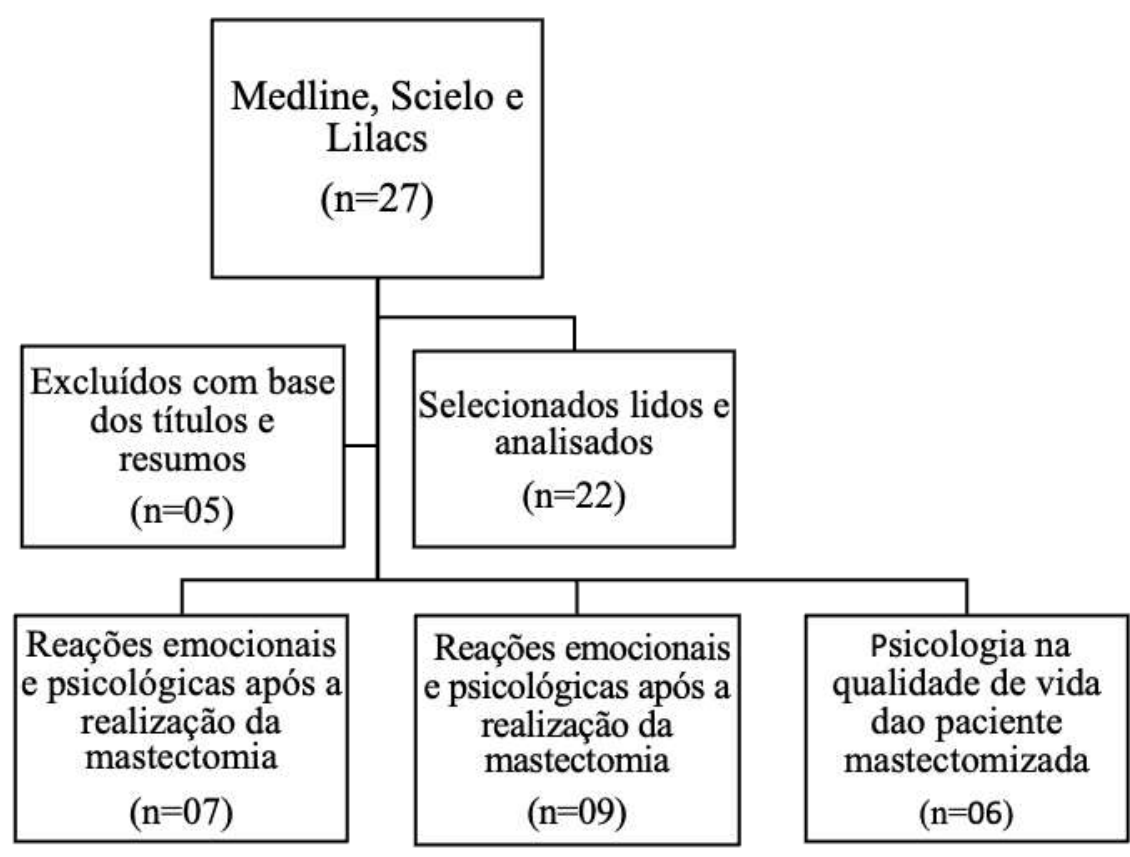

Fonte: Autores.

De acordo com os descritores utilizados na presente pesquisa foram encontrados estudos que contemplam as possíveis importâncias do acompanhamento psicológico em pacientes que realizaram a mastectomia e destacam-se os efeitos psicológicos que acometem pré e após o procedimento da retirada da mama. 
Considerando os objetivos dessa pesquisa, podemos destacar algumas considerações feitas por pesquisadores. De acordo com os estudos encontrados, a mastectomia como forma de tratamento para o câncer de mama sempre será um procedimento cruel para qualquer mulher, onde afeta fisicamente e psicologicamente, sabemos que a feminilidade é algo já muito cultural e considerado importante para a maioria das mulheres assim tento um abalo psicológico maior.

Para Regis e Simões (2005) a mastectomia é um procedimento cirúrgico, cuja finalidade é erradicar a presença local do câncer. A cirurgia é considerada uma das formas de tratamento mais temidas pela mulher, levando a sentimento de tristeza, vergonha e depressão. A cirurgia traz em si um caráter agressivo e traumatizante para a vida da mulher, levando a uma desfiguração e, consequentemente, a uma alteração da autoimagem.

A mastectomia desagrega o funcionamento biopsicossocial, sendo necessária a readaptação da sua vida intrapsíquicas. O prognostico do câncer de mama é geralmente incerto e possui tratamentos agressivos provocando a mutilação e cicatrizes físicas e psicológicas. Se expondo a mudanças na sua imagem corporal que implicam na sua vida diária, social e familiar. (DURA, 2002).

De acordo com Bervian e Girardon-Perlini (2006), a reabilitação de uma mastectomia tem os primeiros meses caracterizados pelo movimento de reorganização para uma reinserção no mundo social, individual e espacial, visto que a mutilação decorrente favorece o surgimento de questões na vida das mulheres, especialmente à imagem corporal. A mulher tem uma forma de percebe e lida com essa nova imagem e como isso afetando sua existência, sendo pontos cruciais para um entender a nova dinâmica que a vida dessas mulheres assume.

Segundo Hopwood et al. (2007), as percepções relativas à imagem corporal em vítimas de câncer de mama incluem insatisfação quanto à anormalidade da aparência, percebida pela perda da integridade e feminilidade, resultando em relutância em olhar para o próprio corpo.

Para Frierson (2006) As emoções negativas e angustiantes ocasionado pela mudança no corpo após o diagnóstico de câncer de mama e da mastectomia representa um estresse relativo. Apresentando manifestações de diversos aspectos como a restrição da exposição do corpo para familiares e principalmente a si mesmo, falta de interesse sexual, irritabilidade, raiva e tristeza ao lembrar-se da mudança no corpo.

Regis (2005) diz que a negação pode ser apresentada como uma forma de defesa por muitas pessoas quando estamos com dificuldades. A mulher com câncer de mama se encontra vulnerável assim tendo esse sentimento com mais intensidade.

Já para Corbellini (2001) muitos dos diagnostico vem como um choque, talvez por não acreditar que o nódulo pode ser algo sério ou porque preferem usar o sentimento de negação para não apresentar os sintomas presentes. Na maioria das vezes a mulher procura adiar o tratamento por causa multiplas, dentre elas a negação de estar com uma doença grave, fator socioeconômico e fatores psicológicos.

Barbosa (2010) Entende-se que o procedimento da mastectomia realizado nas mulheres, provocar sentimento de luto diante da perda da mama. Diante disso essa perda, que atingi um órgão, cuja simbologia tem como a representatividade a própria essência da feminilidade, onde altera o papel de ser mulher e papeis como esposa e mãe, gerando baixa autoestima e tristeza.

De acordo com Covich (2004) diz que após a retirada da mama existem consequências emocionais, que necessitam de assistência adequando com o objetivo de reintegração familiar e social, visando facilitar a adaptação da paciente a sua nova realidade. A mastectomia é de difícil aceitação, ocasionando atrasos e ate mesmo abandono no tratamento, é de suma importância a atuação em conjunta dos membros da equipe de saúde e outros profissionais envolvidos sendo benéfico para a reabilitação.

Silva (2010) destaca que, a família em muitas situações, ficam abaladas com a doença e seus tratamentos onde não tem a capacidade de oferecer apoio de que a mulher necessita. E por isso trazem alguns prejuízos na recuperação da mulher. 
Portanto, entende-se a importância da família ser bem orientada pelos profissionais, a fim de serem capazes de dar o suporte adequado de que as mulheres necessitam

Silva (2010) conclui ainda que o suporte da família representa um fator importância para as mulheres na adaptação e superação diante da doença. Ressalta-se que a união e a relação com a família favorecem um ambiente harmonioso, assim como ajuda emocional, físicas e cuidado as mulheres, já que se encontram impossibilitadas de realizar algumas atividades diante disso o apoio dos familiares e amigos juntos ao tratamento e à reabilitação torna-se fator fundamental, pois a doença permite que a mulher se sinta fragilizada. A importância da participação da família podendo ser demonstrada por meio do apoio emocional, do carinho, da compreensão e até mesmo financeiramente, com isso contribuindo de forma positiva na trajetória da mulher durante o tratamento.

Miceli (2006), diz que o conceito da qualidade de vida está relacionado à saúde, centrado na avaliação subjetiva do paciente, com isso ligado ao impacto da saúde sobre a capacidade do indivíduo para viver plenamente. Dependendo das percepções, sentimentos, expectativas individuais e crenças, variando com o tempo, de acordo com as mudanças ocorridas e com o que está à sua volta, onde envolve dimensões física, psicológica e social, interligadas entre si.

Venâncio (2017) relata que o psicólogo deve ter primeiramente competência técnica e ética para lidar com um sofrimento dessas mulheres. Diante disso, o papel assumido pelo psicólogo com a paciente permitindo que ele mantenha neutralidade o resguardando do sofrimento junto com ela, porem isso não o impede de sentir o sofrimento. Aliás, é justamente o sentimento de sofrimento da paciente que deve impulsionar e motivar este profissional a disponibilizar seus conhecimentos para ajudá-la.

\section{Conclusão}

De acordo com os dados encontrados foram observados que muitas mulheres sofrem na retirada da mama principalmente piscologicamente, com isso mostramos a importância para essa realidade e a necessidade de um acompanhamento psicológico das mesmas, para facilitar de alguma forma um seu tratamento. Por isso, mostramos também a importância de mais trabalhos na área, principalmente com estudos de caso para que isso posso ser comparado e utilizado como base para outros acompanhamentos em outras pacientes.

Se deve demonstrar também que existem formas de reposição (caso seja o desejo das mesmas), como cirurgias plásticas que podem auxiliar auto estima e melhorar assim a qualidade de vida das pacientes. Por isso, reforçamos a grande necessidade de um acompanhamento psicológico durante o tratamento da doença.

\section{Referências}

Alves, M. L. M. et al. (2007). Ansiedade no período pré-operatório de cirurgias de mama: estudo comparativo entre pacientes com suspeita de câncer e a serem submetidas a procedimentos estéticos. Rev. Bras. Anestesiol., Rio de Janeiro, v. 57, n. 2, p. 147-56.

Brasil, Ministério da Saúde Instituto Nacional do Câncer (INCA). (2008). Estimativas de incidência e mortalidade por câncer no Brasil. Jul, 25. http:// www.hospcancer-ICC.org.br/conteudo.php.

Corbellini, V. L. (2001). Câncer de mama: encontro solidário com o temor do desconhecido. Rev. Gaucha Enferm. Porto Alegre, v. 22 , n. 1, p. 42-68.

Covich, J. L. (2000). The relationship between breast reconstruction and psychological adjustment when a mastectomy is necessary. Dissertation Abstracts International: Section B: The Sciences \& Engineering, 60 (12-B), 6356. (University Microfilms International).

Dias, M. R.; Dura, E. (2002). Territórios da psicologia oncológica. Lisboa: Climepsi Editores.

DUARTE, T. P.; ANDRADE, A. C. (2003). Enfrentando a mastectomia: análise dos relatos de mulheres mastectomizadas sobre questões ligadas à sexualidade. Estud Psicol. 8(1):155-63.

Fonseca, A. M. J. S. (1988). Vivência corporal, imagem do corpo e mastectomia. In: Anais da 18 a Reunião Anual de Psicologia; nov. 9-13; Ribeirão Preto, Brasil. Ribeirão Preto (SP): Sociedade de Psicologia de Ribeirão Preto. p.181-89. 
Frierson, G. M. et al. (2006). Body change stress for women with breast cancer: the breast-impact of treatment scale. Ann Behav Med. 32(1): 77-81.

Huguet, P. R, Morais S. S., Osis, M. J. D., Pinto-Neto, A. M., Gurgel, M. S. C. (2009). Qualidade de vida e sexualidade de mulheres tratadas de câncer de mama. Rev Bras Ginecol Obstet 2009; 31(2): 61-7.

Hopwood, P. et al. (2007). The impact of age and clinical factors on quality of life in early breast cancer: an analysis of 2208 women recruited to the UK START Trial. Standardisation of Breast Radiotherapy Trial. Breast, Jun; 16:241-51.

Oliveira, M. M.; Monteiro, A. R. (2004). Mulheres mastectomizadas: ressignificação da existência, Texto Contexto Enferm. Jul-Set; 13(3):401-8.

Pereira, S. G. et al. (2006). Vivências de cuidados da mulher mastectomizada: uma pesquisa bibliográfica. Rev Bras Enferm. 59 (6):791-5.

Perlini, N. et al. (2006). A família (con)vivendo com a mulher/mãe após a mastectomia. Rev Bras Cancerol. 52(2):121-28.

Pinheiro, A. B. (2013). Transtornos psicológicos que afetam a qualidade de vida de mulheres mastectomizadas. Salvador. iv; 39 p. [tab.].

Pinho, L. S. et al. (2007). Câncer de mama: da descoberta à recorrência da doença. Revista Eletrônica de Enfermagem. Jan-Abr; 9(1): 154-165. http://www.fen.ufg.br/revista/v9/n1/v9n1a12.htm157.

Regis, M. F., SimoeS, M. F. (2005). Diagnóstico de câncer de mama, sentimentos, comportamentos e expectativas de mulheres. Revista eletrônica de enfermagem, Rio de Janeiro v. 07 n.01, p. 81 -68.

Regis, M. F. S., Simões, S. M. F. (2005). Diagnóstico de câncer de mama: sentimentos, comportamentos e expectativas de mulheres. Revista Eletrônica Enfermagem. 7(1): 81-6.

Rodrigues, N. S. et al. (2017). Importância do acompanhamento psicológico em mulher mastectomizada: artigo de revisão. Arq. Catarin. Med. São Paulo, v. 46 , n. 1, p. 164-172, jan/mar.

Sampaio, A. C. P. (2006). Mulheres com câncer de mama: análise funcional do comportamento pós-mastectomia. Tese de Mestrado, Universidade Católica de Campinas. Acesso em 18.03.20. http://dominiopublico.mec.gov/download/texto/cp000360.pdf

Stanton, A. L. et al. (2005). Promoting adjustment after treatment for cancer. Cancer. v. 104, n. 11, p. $2608-13$.

Talhaferro, B. et al. (2007). Mastectomia e suas conseqüências na vida da mulher. Arq Ciênc Saúde. 14(1): 412-18

Venâncio, J. L. (2004). Importância da Atuação do Psicólogo no Tratamento de Mulheres com Câncer de Mama. Rev. Bras. Cancerol. Rio de Janeiro, v. 50, p. $55-63$.

Estrela, C. (2018). Metodologia Científica: Ciência, Ensino, Pesquisa. Editora Artes Médicas. 